\title{
ORIENTACIÓN NUTRICIONAL SOBRE EL CONSUMO ADECUADO DE BEBIDAS EN ESCOLARES
}

\author{
NUTRITIONAL GUIDANCE ONTHE APPROPRIATE CONSUMPTION OF BEVERAGES IN SCHOOLS
}

\author{
García Badillo Paola Estefanía ${ }^{1}$, Noyola Pescina Tania ${ }^{1}$, Hernández Blanco María Lourdes ${ }^{2}$, Peralta \\ Suchil Julieta Nataly ${ }^{3}$
}

1 Universidad Autónoma de San Luis Potosí, Facultad de Enfermería y Nutrición, Licenciatura en Nutrición. 2 Universidad Autónoma de San Luis Potosí, Facultad de Enfermería y Nutrición, Unidad de Cuidados Integrales e Investigación en Salud. 3 Universidad Autónoma de San Luis Potosí, Facultad de Enfermería y Nutrición, Maestría en

Salud Pública

Citation: García Badillo PE., Noyola Pescina T., Hernández Blanco ML, Peralta Suchil JN. (2017) Orientación nutricional sobre el consumo adecuado de bebidas en escolares, Revista de Salud Pública y Nutrición, 16(2), 19-27. Editor: Esteban G. Ramos Peña, Dr. CS., Universidad Autónoma de Nuevo león, Facultad de Salud Pública, Monterrey Nuevo León, México.

Copyright: (2017 García Badillo PE. et al. This is an open-access article distributed under the terms of Creative Commons Attribution License [CC BY-ND 4.0], which permits unrestricted use, distribution, and reproduction in any medium, provided the original author and source are credited.

Competing interests: The authors have declared that no competing interests exist.

DOI: https://doi.org/10.29105/respyn16.2-3

*Email: paoegb@hotmail.com 


\title{
ORIENTACIÓN NUTRICIONAL SOBRE EL CONSUMO ADECUADO DE BEBIDAS EN ESCOLARES
}

\author{
García Badillo Paola Estefanía ${ }^{1}$, Noyola Pescina Tania ${ }^{1}$, Hernández Blanco María Lourdes ${ }^{2}$, Peralta Suchil \\ Julieta Nataly ${ }^{3}$
}

\begin{abstract}
1 Universidad Autónoma de San Luis Potosí, Facultad de Enfermería y Nutrición, Licenciatura en Nutrición. 2 Universidad Autónoma de San Luis Potosí, Facultad de Enfermería y Nutrición, Unidad de Cuidados Integrales e Investigación en Salud. 3 Universidad Autónoma de San Luis Potosí, Facultad de Enfermería y Nutrición, Maestría en Salud Pública.
\end{abstract}

\begin{abstract}
RESUMEN
Introducción: Los principales tipos de bebidas que contribuyen con el mayor aporte energético en los escolares son las bebidas azucaradas, sin embargo, su ingesta es mayor a la recomendada y sin tomar en cuenta la del agua simple al día. Estos hábitos pueden contribuir a ganancia de peso ponderal y a padecer enfermedades crónicas. Objetivo: Ejecutar un plan de orientación nutricional sobre el consumo de bebidas en escolares de educación primaria. Métodos: Estudio descriptivo con 56 escolares de educación primaria. Se realizó en tres fases, primero se realizó antropometría, se determinó el conocimiento y frecuencia de consumo de bebidas, después cinco sesiones de orientación nutricional y finalmente se reevaluó la primera fase; se utilizó estadística descriptiva, para el procesamiento se utilizó SPSS v 21. Resultados: 56 niños del estudio, $51.8 \%$ eran mujeres, $48.2 \%$ hombres, en edad promedio de 8.1 años DE. $0.908,25 \%$ aprobó el cuestionario de conocimientos previo a las sesiones, $57 \%$ tomaban agua natural, el resto eran bebidas azucaradas, que consumían hasta más de 5 veces a la semana; posterior a la orientación nutricional el $32 \%$ incrementaron conocimiento $(p=0.007), 76.5 \%$ tomaban agua natural. Conclusiones: Se muestra una ejecución positiva del plan de orientación nutricional, se incrementó el conocimiento, sin embargo, debido a la influencia familiar sobre los hábitos alimenticios hacia los escolares no se encontró una destacada disminución en las bebidas azucaradas. Se sugiere llevar a cabo una intervención junto con los padres de familia en un periodo y muestra mayor para incrementar efectividad.
\end{abstract}

Palabras Clave: salud del niño, ingestión de líquidos, agua bebible.

\section{ABSTRACT}

Introduction: The main types of drinks that contribute the major energy supply in schoolchildren are sugary drinks, however, their intake is higher than recommended and without taking in count the one of the simple water at day. These habits may contribute to weight gain and to chronic diseases. Objective: Perform a plan of a nutritional orientation on the consumption of beverages in elementary school students. Methods: Descriptive study with 56 primary school children. It was performed in three phases, first anthropometry was performed, the knowledge and frequency of consumption of drinks were determined, then five sessions of nutritional orientation and finally the first phase was reevaluated; descriptive statistics were used, SPSS v 21 was used for processing. Results: 56 children in the study, $51.8 \%$ were women, $48.2 \%$ were men, with a mean age of 8.1 years SD. $0.908,25 \%$ passed the knowledge questionnaire prior to the sessions, $57 \%$ ingest natural water, the rest were sugary drinks, which consumed up to more than 5 times a week; After nutritional counseling, $32 \%$ increased knowledge $(p=0.007)$, $76.5 \%$ ingest natural water. Discussion: It shows a positive performance of nutritional orientation, increased knowledge, however, due to the environmental influence on eating habits towards schoolchildren did not find an outstanding decrease in sweetened beverages. It is suggested to carry out an intervention together with the parents in a larger period and a bigger sample for increase effectiveness.

Key words: child health, fluid intake, drinking water. 


\section{Introducción}

El agua es un compuesto esencial para la vida, constituye de 50 a $75 \%$ del peso de un individuo, aunque esto depende de la edad, el sexo y la composición corporal. El ser humano obtiene de 70 a $80 \%$ de su requerimiento hídrico de las bebidas y de 20 a $30 \%$ de los alimentos que consume.

Los principales tipos de bebidas que contribuyen con el mayor aporte energético en la población mexicana son: refrescos, bebidas elaboradas con jugos de fruta (con o sin azúcar), aguas frescas y jugos elaborados con $100 \%$ de fruta a los que se agrega azúcar, esto representa la quinta parte del valor energético total (VET) ingerido por los mexicanos, y constituyen un factor importante en el aumento de peso. Así como la Encuesta Nacional de Salud y Nutrición (ENSANUT) 2006, en la frecuencia de consumo de alimentos reportó que se consumían 17 tipos de bebidas, entre ellas las azucaradas, de éstas el $20.7 \%$ correspondía a la energía total consumida. Así mismo, reportó que se incrementó un $12 \%$ el consumo de refrescos con respecto a 1989, por lo tanto, a nivel mundial México se ubicó como el segundo país con mayor consumo de refresco y para 2012 ocupó el primer lugar (Hernández et al., 2012).

$\mathrm{Al}$ respecto, $28.1 \%$ del consumo total de bebidas en los niños mexicanos durante el día es de agua simple; $44 \%$ lo complementan con bebidas azucaradas y el resto de líquidos proviene de los alimentos (Irizarry, 2009). Lo anterior lleva a que un $50 \%$ de los niños mexicanos no alcancen la recomendación mínima del consumo de agua simple al día de 1.8 litros (Hernández et al., 2012).

En relación a la inadecuada ingesta de bebidas azucaradas sumada a otros factores, en la última década en México, la prevalencia de sobrepeso y obesidad en escolares ha aumentado $42 \%$ (Carriedo et al., 2013), así como ENSANUT 2012 reporta ésta prevalencia en $34.4 \%$, de igual manera en consecuencia de éstas cifras, se aumenta el riesgo a presentar enfermedades crónico degenerativas, por lo que el Hospital Infantil de México en 2006 creó un modelo predictivo matemático a fin de estimar la carga económica y en salud de la obesidad en niños mexicanos durante los años 2006-2050 y se demostró por resultados que en 2015 se presentarían los primeros casos de diabetes mellitus $2 \mathrm{y}$ de hipertensión arterial que pueden ser prevenibles. En
2012 la Secretaria de Salud informó que invierte en la atención de la obesidad y sus complicaciones 42 mil millones de pesos anuales, así como las pérdidas por productividad (Acosta et al., 2002).

Ante tal situación, la Secretaría de Salud (2010), estableció el Acuerdo Nacional para la Salud para promover el aumento de la disponibilidad, accesibilidad y el consumo de agua simple potable y disminuir el consumo de azúcares y grasas en bebidas.

La alimentación y la nutrición son procesos influenciados por aspectos biológicos, ambientales y socioculturales. Durante la infancia, éstos contribuyen a un desarrollo y crecimiento óptimo, así como una maduración biopsicosocial, por lo que es necesario que los niños adquieran durante esta etapa hábitos alimentarios saludables y conductas que perduren en el tiempo (Araneda, Bustos, Cerecera y Amigo, 2015). En este sentido, la escuela se considera un lugar adecuado para la promoción de estilos saludables, por ello la Secretaría de Educación Pública (SEP) estableció lineamientos para la venta de alimentos y bebidas en los planteles de educación básica (Macías, Gordillo y Camacho, 2012). Así como Kaushik, Mullee, Bryant y Hill (2007) sugieren que es importante que en México se garantice el acceso libre al agua potable dentro de todas las escuelas y se regule la publicidad de bebidas azucaradas dirigida a los niños, debido a que se ha encontrado en estudios que un acceso limitado al agua natural resulta en una inadecuada ingesta del agua en las escuelas.

La iniciativa de una orientación nutricional es funcional debido a que los niños pasan varias horas del día en la escuela, lugar adecuado para la promoción de estilos de vida saludables y recibir orientación sobre la ingesta adecuada de agua natural que puede disminuir la de bebidas azucaradas (Hernández et al., 2012).

Ante lo antes expuesto, el centro de salud comunitario de la Universidad Autónoma de San Luis Potosí, denominado Unidad de Cuidados Integrales en Investigación en Salud (UCIIS) que es un espacio destinado para que los Pasantes de la Licenciatura en Enfermería y Nutrición puedan desenvolverse profesionalmente, implantó una estrategia de salud escolar en escuelas de educación 
básica de la comunidad en la que se encuentra, por lo tanto, una de ellas es la Escuela Primaria "José Mariano Jiménez", ubicada al oriente del municipio de San Luis Potosí, en donde por medio de una iniciativa educativa de orientación nutricional se pretendió contestar la pregunta ¿Habrá una disminución en la frecuencia de consumo de bebidas azucaradas y un aumento en el conocimiento teórico sobre consumo de bebidas por medio de una orientación nutricional en escolares de educación primaria?

Teniendo en cuenta lo anterior, el presente artículo se planteó el objetivo de aumentar el conocimiento teórico sobre el consumo de bebidas y disminuir la frecuencia de consumo de bebidas azucaradas por medio de una orientación nutricional en escolares de educación primaria. Además, se incluyó el objetivo específico de conocer y comparar el estado nutricio de los escolares previo y posterior a la orientación nutricional.

Por lo tanto, estudio se desarrolló a partir de la hipótesis, que una orientación nutricional aumentará el conocimiento teórico sobre el consumo de bebidas y disminuirá la frecuencia de consumo de bebidas azucaradas en escolares.

\section{Material y Métodos}

Estudio longitudinal, cuasi experimental que involucra la participación de 56 niños de segundo a sexto grado de primaria con edad de 7 a 12 años, de una población total de 545 alumnos.

Como criterio de inclusión, se requirió la firma de un consentimiento informado por parte de los padres de familia y que los estudiantes estuvieran inscritos en la Escuela Primaria José Mariano Jiménez, ubicada en la colonia La Libertad, San Luis Potosí, S.L.P.

El criterio de exclusión fue la negación de la firma del consentimiento informado por los padres de familia o la participación de los alumnos negada por los docentes a su cargo que mantuvieron como prioridad lograr cubrir el programa educativo en tiempo y forma.

Por otro lado, el criterio de eliminación, que descartó a siete alumnos, fue la ausencia a sesiones de orientación nutricional y la falta de información requerida para la evaluación de los escolares.
Éste estudio se realizó en tres fases en un periodo de cinco meses durante el turno matutino, la primera consistió en generar un diagnóstico nutricional y de conocimientos sobre el consumo de bebidas en escolares dentro de la biblioteca de la escuela, para ello se tomaron medidas antropométricas para la obtención de un diagnóstico nutricio inicial, analizando el Índice de Masa Corporal para la Edad con las tablas del Center of Desease Control and Prevention (CDC), 2000 (IMC/Edad) y posteriormente se aplicó el cuestionario "Conocimientos sobre bebidas" (ENSANUT, 2006) con 9 preguntas de opción múltiple con temas sobre la recomendación de ingesta del agua simple, sus beneficios y los riesgos de consumir bebidas azucaradas, evaluado en una escala del 0 al 10 siendo la calificación aprobatoria de 6 a 10 y reprobatoria de 0 a 5.9, aunado a ello se realizó una "Frecuencia de consumo de bebidas", en donde los escolares marcaban en una escala de frecuencia de consumo de cuatro bebidas (agua sola, refresco, jugo industrializado y agua de frutas con azúcar), cuyas opciones de elección fueron: "nunca", "una a cuatro veces a la semana" y "cinco o más veces a la semana" para conocer los hábitos de ingesta de los participantes.

En la segunda fase, se llevó a cabo la orientación nutricional en los escolares con cinco sesiones cada una duración de entre 15 minutos y 1 hora, realizándolas entre cada dos y tres semanas aproximadamente, mediante talleres interactivos con temas como: la importancia del consumo de agua natural, las consecuencias de la ingesta de bebidas azucaradas, el requerimiento de consumo de agua natural y preparación de bebidas bajas en calorías. Se trabajó en la promoción del consumo adecuado de bebidas en el ambiente escolar por medio de la decoración de garrafones en los salones de clases de los participantes, la creación de un mural y un juego de "El Avión" en forma de botella de agua en un área de juego.

Por último, en la tercera fase, se evaluaron los resultados obtenidos posteriores a las sesiones educativas de orientación nutricional, mediante las medidas antropométricas obteniendo un diagnóstico nutricio, el cuestionario de conocimientos y la frecuencia de consumo de bebidas. 
Para el análisis estadístico, se utilizó estadística descriptiva, con frecuencias absolutas y relativas, así como con medidas de tendencia central y de dispersión; así mismo, se corrió la prueba no paramétrica de los rangos con signo de Wilcoxon para los resultados del cuestionario de conocimientos y el diagnóstico nutricio de los escolares. Por otro lado, se utilizó la prueba de Bowker McNemar para los resultados de la frecuencia de consumo de bebidas. En todas las pruebas mencionadas se consideró un nivel de significancia de $\mathrm{p}<0.05$, con apoyó del paquete estadístico SPSS v 21.

Manteniendo los principios éticos, el presente estudio se apegó al artículo 100 de la Ley General de Salud en Materia de Investigación de Salud (Secretaría de Salud, 1987). Previo al desarrollo del estudio se solicitó y se obtuvo el registro CEIFE2016-169 ante el Comité de Ética en Investigación de la Facultad de Enfermería y Nutrición de la Universidad Autónoma de San Luis Potosí, aunado a ello se informó a las autoridades escolares y padres de familia al firmar el consentimiento informado sobre el desarrollo y los resultados del estudio; así como se acordó que no existe conflicto de intereses entre los profesionales de la salud que llevaron a cabo la investigación.

\section{Resultados}

Características de la población de estudio

De los 56 escolares participantes en el estudio, $51.8 \%$ eran mujeres y $48.2 \%$ hombres, la media de la edad fue de $8.1 \pm 0.908$ (DE) años.

Nivel de conocimientos sobre bebidas

En la evaluación inicial del conocimiento sobre el adecuado consumo de bebidas, se encontró que $75 \%$ de los escolares obtuvieron una calificación reprobatoria en una escala del 0 al 5.9, y $25 \%$ fueron aprobatorios en una escala del 6 al 10 .

Posteriormente a las sesiones de orientación nutricional en dónde se explicó dinámica y participativamente los temas del cuestionario de conocimientos, se encontró una notable mejoría por un incremento al $57 \%$ de niños con calificaciones aprobatorias y disminución con calificaciones reprobatorias $(\mathrm{p}=0.012)$, (Ver tabla 1$)$.
Tabla 1. Escolares por momento de orientación según calificación de examen de conocimientos

\begin{tabular}{lcrcr}
\hline \multirow{2}{*}{ Calificación } & \multicolumn{2}{c}{ Antes } & \multicolumn{3}{c}{ Después } \\
& Frecuencia & $\%$ & Frecuencia & $\%$ \\
\hline Aprobatoria & 14 & 25 & 32 & 57 \\
Reprobatoria & 42 & 75 & 24 & 43 \\
Total & 56 & 100 & 56 & 100 \\
\hline
\end{tabular}

Fuente: Directa

Frecuencia de consumo de bebidas

Se encontró en primera instancia que más del $50 \%$ de los niños encuestados consumían refrescos, jugos industrializados y agua de frutas endulzada de una a cuatro veces a la semana y el $57 \%$ consumían más de 5 veces a la semana agua natural. Posterior a éstos resultados, se desarrollaron las sesiones de orientación nutricional con una metodología interactiva para hacer consciente al niño sobre los beneficios del consumo de agua natural y la disminución de bebidas azucaradas, en las cuales los niños mostraron interés participando activamente. Una vez concluidas éstas sesiones se observó un incremento del $19.5 \%$ el consumo de agua natural más de 5 veces por semana (ver tabla 2), sin embargo, no resultó en un valor significativo la comparación de frecuencias $(\mathrm{p}=0.087)$ mostrado en la Tabla 3. Por otro lado, se redujo a menos del 50\% las bebidas azucaradas con frecuencia de una a cuatro veces por semana, se incrementó al 50\% los escolares que no toman refresco a la semana (Tabla 2) y además se obtuvo un valor significativo en la comparación de frecuencias de éstas bebidas $(\mathrm{p}=0.001)$ reflejado en la Tabla 4 . Con lo antes mencionado, se descarta la hipótesis nula y se aprueba la alterna, al demostrar que disminuyó la frecuencia de consumo de bebidas azucaradas en escolares posterior a la orientación nutricional. 
Tabla 2. Escolares ${ }^{\star \&}$ por frecuencia consumo bebidas semanal pre y post orientación nutricional según tipo de bebida

\begin{tabular}{llllllc}
\hline \multirow{2}{*}{ Bebida } & \multicolumn{3}{c}{ Pre orientación } & \multicolumn{3}{c}{ Post orientación } \\
& Nunca & 1 - 4 veces & Más de 5 veces & Nunca 1 - 4 veces & Más de 5 veces \\
\hline Agua Natural & 5,5 & 37,5 & 57,0 & 3,5 & 20,0 & 76,5 \\
Refresco & 20,0 & 62,5 & 18,5 & 50,0 & 43,0 & 7,0 \\
Jugo Ind. & 20,0 & 52,0 & 28,0 & 32,0 & 48,0 & 20,0 \\
Agua de frutas & 11,0 & 68,0 & 21,0 & 39,0 & 41,0 & 20,0 \\
\hline
\end{tabular}

*En porcentajes; \& $\mathrm{n}=56$

Tabla 3. Comparación entre frecuencia de consumo de "agua sola" pre y post orientación nutricional

\begin{tabular}{|c|c|c|c|c|c|c|c|c|c|c|}
\hline & \multirow{3}{*}{$\begin{array}{l}\text { Frecuencia de } \\
\text { consumo }\end{array}$} & \multicolumn{7}{|c|}{ Post-Orientación } & \multirow{2}{*}{\multicolumn{2}{|c|}{ Total }} \\
\hline & & \multicolumn{2}{|c|}{ Nunca } & \multicolumn{3}{|c|}{ 1-4 veces } & \multicolumn{2}{|c|}{ Más de 5 veces } & & \\
\hline & & Núm. & $\%$ & Núm. & & $\%$ & Núm. & $\%$ & Núm. & $\%$ \\
\hline \multirow{4}{*}{$\begin{array}{c}\text { Pre- } \\
\text { Orientación }\end{array}$} & Nunca & 1 & 33.3 & & 1 & 33.3 & 1 & 33.3 & & 3100.0 \\
\hline & $1-4$ veces & 1 & 4.8 & & 6 & 28.6 & 14 & 66.7 & & 21100.0 \\
\hline & Más de 5 veces & 0 & 0.0 & & 4 & 12.5 & 28 & 87.5 & & 32100.0 \\
\hline & Total & 2 & 3.6 & & 11 & 19.6 & 43 & 76.8 & & 56100.0 \\
\hline
\end{tabular}

Fuete: Directa "Frecuencia de consumo de bebidas"

$P>0.05$

Tabla 4. Escolares ${ }^{\&}$ según frecuencia de consumo de bebidas azucaradas ${ }^{\star}$ pre y post orientación nutricional

\begin{tabular}{cccccc}
\hline & & \multicolumn{3}{c}{ Post-Orientación } & \multirow{2}{*}{ Total } \\
\cline { 3 - 5 } Frecuencia de consumo & Nunca & $1-4$ veces & Más de 5 veces & \\
\cline { 3 - 6 } & Nunca & 7.1 & 1.8 & 0.0 & 8.9 \\
Pre- & $1-4$ veces & 30.3 & 39.3 & 5.4 & 75.0 \\
Orientación & Más de 5 veces & 1.8 & 12.5 & 1.8 & 16.1 \\
& Total & 39.3 & 53.6 & 7.1 & 100.0 \\
\hline
\end{tabular}

Fuente: Directa "Frecuencia de consumo de bebidas"

$P<0.05, n=56$

* Las "bebidas azucaradas" incluyen la evaluación de: refresco, jugo industrializado y agua de frutas con azúcar.

¿En porcentajes 


\section{Diagnóstico nutricio por IMC/ Edad}

La tabla 5 muestra los porcentajes de los niños estudiados según diagnóstico por IMC llevado a cabo por medidas antropométricas. El mayor porcentaje de presenta en IMC normal en ambos momentos de la orientación alimentaria. No se encontró diferencia entre el IMC/ Edad pre y post la orientación nutricional, $(\mathrm{p}=0.414)$.

Tabla 5. Porcentaje de escolares según diagnóstico nutricio por IMC/ Edad pre y post orientación nutricional presentada en porcentajes

\begin{tabular}{lcc} 
Diagnóstico Pre orientación & Post orientación \\
\hline Bajo Peso & 0 & 1.8 \\
Normopeso & 64.3 & 60.7 \\
Sobrepeso & 17.9 & 16.1 \\
Obesidad & 17.9 & 21.4 \\
Total & 100.0 & 100.0 \\
Fuente: Directa "Diagnóstico nutricio" \\
P $>0.05$ \\
$n=56$
\end{tabular}

\section{Discusión}

Los resultados de este estudio muestran que la iniciativa de la estrategia de orientación nutricional fue favorable, debido a que se incrementó el conocimiento en aproximadamente la mitad de escolares $(\mathrm{P}=0.012)$. Este incremento en los conocimientos de los escolares es semejante a lo reportado en un estudio por Rodríguez et. al. 2013, en el cual encontraron una tendencia a la mejoría de conocimientos nutricionales tras una intervención educativa moderada pero específica (aplicada por un profesional de la nutrición y no por sus profesores) sobre nutrición y hábitos de Vida Saludable, a pesar de que los niños estudiados partían de una base de conocimientos buena. Esto nos lleva a pensar que tras una intervención educativa impartida por profesionales sanitarios se puede producir un mayor impacto y una mejoría en los conocimientos nutricionales.

Los escolares por su corta edad no están conscientes de los riesgos a su salud que causa una alta ingesta de azúcares simples y sus elecciones son influenciadas ambientalmente, como en la escuela se observó que comúnmente madres de familia en el recreo les proporcionaban a los escolares bebidas azucaradas, aportando únicamente calorías sin nutrientes (Rivera et al., 2008).

En cuanto a las medidas antropométricas, se tomaron en forma de monitoreo por el corto tiempo de duración de la misma por lo que no se observaron cambios significativos $(\mathrm{p}=0.414)$ en el diagnóstico nutricio de los niños evaluados, ya que no se evaluó el factor de la ingesta de alimentos. Lo que coincide con estudios que muestran que cuando los objetivos son de aprendizaje relacionados con la nutrición, el éxito es mayor que cuando el objetivo es reducir el IMC, aun cuando la intervención dure meses o incluso años (Flores, Klünder y Medina, 2008), como James et al. (2004) menciona que al disminuir su consumo de bebidas azucaradas después de 12 meses disminuyó $0.2 \%$ sobrepeso y obesidad.

En los últimos años, resultados de diversos estudios han señalado que los niños que consumen mayores cantidades de bebidas azucaradas tienen $55 \%$ más probabilidades de tener un riesgo para la salud, como lo es el sobrepeso y la obesidad a diferencia de aquellos que consumen menos (Velasco et al., 2015). En los escolares participantes, previamente a la orientación nutricional, se reflejaron hábitos en preferencia a bebidas azucaradas y poco conocimiento sobre las bebidas, como resultó en la evaluación inicial de este estudio, obteniendo un promedio de calificación de 5.1, situación que indica que el niño no tiene asociación de las bebidas con su salud, estos resultados coinciden con un estudio realizado por Théodore et al. (2011) los niños eligen las bebidas (dulces por lo general) principalmente en función de sus gustos y preferencias de sabor y no a raíz de consideraciones relacionadas con su salud. Estos resultados coinciden con los hallazgos de un estudio realizado por el Departamento de Nutrición y Bioquímica de Bogotá en 2014 con niños de siete y ocho años, donde se concluye que cuando los niños gozan de libertad para seleccionar alimentos y bebidas, su elección no está dictada por atributos para su salud sino por sus preferencias gustativas.

En ocasiones la población está informada y conoce los conceptos básicos nutricionales, sin embargo, en la mayoría de los casos no se ponen en práctica por no comprender el impacto que puede llegar a causar en ellos, por lo que se debe afrontar la enseñanza desde una perspectiva multifactorial. En la literatura señala que las intervenciones a nivel escolar 
constituyen una de las estrategias más importantes para enfrentar la obesidad en la población infantil, ya que los primeros años de enseñanza básica constituyen un periodo de desarrollo de hábitos de alimentación por su población cautiva y vulnerable a los beneficios de la educación. A pesar de numerosos estudios, al momento no se tiene una estrategia universal aplicable en las escuelas (Flores, Klünder y Medina, 2008 y Ratner et al., 2013).

De los resultados del cuestionario de frecuencia de consumo de bebidas aplicados posteriormente a la orientación nutricional, fueron favorables, debido a la disminución de consumo de bebidas azucaradas (refrescos, jugos industrializados y agua de frutas endulzadas con azúcar) con un valor significativo ( $\mathrm{p}=$ 0.001). En cambio, el incremento de consumo de agua natural no tuvo significancia $(\mathrm{p}=0.087)$ al igual que el diagnóstico nutricio $(\mathrm{p}=0.414)$. Sin embargo, en estudios similares respecto a la disminución de bebidas azucaradas, los resultados fueron poco efectivos para reducir peso, argumentando que el hogar es además de la escuela otro lugar significativo donde hay un importante consumo de estas bebidas, factor que no ha sido controlado suficientemente en previas investigaciones (Hernández et al., 2012), por lo que se considera como factor importante para cambiar los hábitos de los escolares, incluir en gran medida la participación de los padres (Lindsay, Sussner y Gotmaker, 2006 y Nickelson, Roseman y Forthofer, 2010).

Por otro lado, es necesario considerar la influencia familiar por ser el primer contacto de la generación de hábitos y conductas de elección relacionadas con la alimentación de los niños, ya que estos son el resultado de una construcción social y cultural acordada implícitamente por sus integrantes (Macías, Gordillo y Camacho, 2012). Sin embargo, en el presente estudio se tuvo como limitación la autorización de padres de familia al no firmar el consentimiento informado para tener una mayor muestra de estudio, por lo que en futuros estudios se tiene que concientizar y promover la participación tanto de los padres de familia como de los maestros para poder incluirlos dentro de las mismas actividades y evaluaciones

\section{Conclusiones:}

Se muestra que la ejecución de un plan de orientación nutricional sobre el consumo de bebidas en escolares fue positivo, ya que posteriormente a ésta, se incrementó el conocimiento sobre bebidas con valor significativo $(\mathrm{p}=0.012)$ y se disminuyó el consumo de bebidas azucaradas $(\mathrm{p}=0.001)$, dando resolución a la pregunta de investigación. En base a lo antes mencionado, se demuestra el objetivo y se acepta la hipótesis alterna: una orientación nutricional aumentará el conocimiento teórico sobre el consumo de bebidas y disminuirá la frecuencia de consumo de bebidas azucaradas en escolares. Sin embargo, no se encontró un aumento significativo en el consumo de agua sola $(\mathrm{p}=0.087)$, así como no se observaron cambios significativos en pre y post diagnóstico nutricio de los escolares $(\mathrm{p}=0.414)$.

Finalmente, se sugiere para tener resultados más favorables, se lleve a cabo en próximas orientaciones o intervenciones nutricionales la inclusión de los padres de familia de escolares de nuevo ingreso a la primaria, por ser el primer nivel de educación donde se empieza a mandar o llevar alimentos y bebidas para ingerir dentro de la institución. Agregando la sugerencia de invitar a los docentes para fomentar la promoción de la ingesta de agua natural dentro y fuera del salón, realizando actividades con los escolares para que tomen conciencia y relacionen la ingesta de bebidas con su salud, todo esto en un periodo y con una muestra mayor para incrementar efectividad.

\section{Agradecimientos:}

Se agradece a la directiva de la Escuela Primaria José Mariano Jiménez, San Luis Potosí, S.L.P. por permitir llevar a cabo el proyecto de investigación con los estudiantes dentro de sus instalaciones.

Se agradece a la Unidad de Cuidados Integrales e Investigación en Salud de la Universidad Autónoma de San Luis Potosí por su apoyo.

Se agradece a la M Admon. Rosa María Guadalupe Andrade Cepeda, Jefa de la Unidad de Posgrado y docente de la Facultad de Enfermería y Nutrición de la Universidad Autónoma de San Luis Potosí, por su apoyo en la revisión del presente artículo.

\section{Bibliografía}

Araneda, J., Bustos, P., Cerecera, F., \& Amigo, H. (2015). Ingesta de bebidas azucaradas analcohólicas e índice de masa corporal en escolares chilenos. Salud Pública México, Vol. 57 (2), pp. 128-134. 
Barquera, S., et. al. (2010). Acuerdo Nacional para la Salud Alimentaria Estrategia contra el sobrepeso y la obesidad. Noviembre 20, 2015, de Secretaría de Salud. Sitio web: http://www.google.com.mx/url?sa=t\&rct=j\&q=\&esrc $=$ s\&source $=$ web $\& \mathrm{~cd}=3 \&$ ved $=0$ ahUKEwiUyonE8O QAhVCyVQKHSLKDDYQFggiMAI\&url=http\%3A \%2F\%2Fwww.promocion.salud.gob.mx\%2Fdgps $\% 2$ Fdescargas $1 \% 2$ Fprogramas\%2FAcuerdo $\% 2520$ Origi nal\%2520con $\% 2520$ creditos $\% 252015 \% 2520 \mathrm{feb} \% 252$ 010.pdf\&usg=AFQjCNGb9ISe46nerZEP2IUPPKLA w1mwjA\&sig2=yAKX2WNAEXP_I2prUFsWlA\&bv $\underline{\mathrm{m}=\text { bv. } 141320020, \mathrm{~d} . \mathrm{cGw}}$

Carriedo, Á., Bonvecchio, A., López, N., Morales, M., Mena, C., Théodore, F., et.al. (2013). Uso del mercadeo social para aumentar el consumo de agua en escolares de la Ciudad de México. Consultado el Septiembre 17, 2015, de Salud Pública Sitio web: http://bvs.insp.mx/rsp/articulos/articulo.php?id=0029 07

Departamento de Nutrición y Bioquímica. Grupo de Investigación Alimentos, Nutrición y Salud. Facultad de Ciencias. Pontificia Universidad Javeriana. Bogotá, Colombia. (2014). Factores influyentes en el comportamiento alimentario infantil. Septiembre 20, 2016, de SCIELO. Sitio web: http://www.scielo.org.co/scielo.php?script=sci_arttext \&pid=S0120-00112014000200010

Flores S., Klünder M., Medina P. (2008). La escuela primaria como ámbito de oportunidad para prevenir el sobrepeso y la obesidad en los niños. Septiembre 18,2016, de SCIELO. Sitio web: http://www.scielo.org.mx/scielo.php?script=sci_arttex $\underline{\mathrm{t} \& \mathrm{pid}=\mathrm{S} 1665-11462008000600018}$

Gutiérrez, J., Rivera-Dommarco, J., Shamah-Levy, T., Villalpando-Hernández, S., Franco, A., CuevasNasu, L., Romero-Martínez, M. \& Hernández-Ávila, M. (2012) Encuesta Nacional de Salud y Nutrición. Instituto Nacional de Salud Pública, Resultados Nacionales. Cuernavaca, México.

Hernández, L., Stern, D., Tolentino, L., Espinosa, J., \& Barquera, S. (2012). Consumo de agua en la población infantil y adolescente. Consultado el Septiembre 17, 2015, de Instituto Nacional de Salud Pública. Sitio web: http://www.h4hinitiative.com/sites/default/files/ basicpage/file/consumoagua_en_ninos_y_adolescente s_insp.pdf

Irizarry, L. (2009). Asociación entre el patrón de consumo de bebidas y la obesidad y sobrepeso en niños mexicanos. Reporte interno. Ciudad de México.
James, J., Thomas, P., Cavan, D., \& Kerr, D. (2004). Preventing childhood obesity by reducing consumption of carbonated drinks: cluster randomised controlled trial. BMJ: British Medical Journal,

328(7450), $1237 . \quad$ Sitio web: http://doi.org/10.1136/bmj.38077.458438.EE

Kaushik, A., Mullee, M., Bryant, T. \& Hill, C. (2007) A study of the association between children's access to drinking water in primary schools and their fluid intake: can water be 'cool' in school. Journal Compilation;33; 33(4):409-415.

Lindsay,A., Sussner, K., Kim, J. \& Gortmaker, S.(2006) The role of parents in preventing childhood obesity. Future Child;16(1):169-86

Macias, A., Gordillo, L., \& Camacho, E. (2012, septiembre). Hábitos alimentarios de niños en edad escolar y el papel de la educación para la salud. Revista Chilena de Nutrición, Vol. 39 (3), pp.40-43.

Nickelson, J., Roseman, M. \& Forthofer, M. (2010) Associations between parental limits, school vending machine purchases, and soft drink consumption among Kentucky middle school students.J Nutr Educ Behav. Mar-Apr;42(2):115-22.

Olaiz-Fernández G, Rivera-Dommarco J, Shamah-Levy T, Rojas R, Villalpando-Hernández S, HernándezAvila M, Sepúlveda-Amor J. (2006). Encuesta Nacional de Salud y Nutrición 2006. Consultado el Septiembre 17, 2015, de Instituto Nacional de Salud Pública. Sitio web:

http://www.google.com.mx/url?sa=t\&rct=i\&q=\&esrc $=$ s\&source=web\&cd=1\&sqi $=2 \& v e d=0$ ahUKEwiZ19P i9e_QAhVJslQKHb4bDjUQFggZMAA\&url=http\%3 A\%2F\%2Fensanut.insp.mx\%2Finformes $\% 2$ Fensanut 2006.pdf\&usg=AFQjCNHrd51rK SP208QwQMul3at UTSuQ\&sig2=xy_g4FCq3gGBT4QGgSzmFA\&bvm $=$ bv.141320020,d.cGw

Pino J.L., López M., Cofre M., González C., Reyes L. (2010). Conocimientos alimentario-nutricionales y estado nutricional de estudiantes de cuarto año básico según establecimientos particulares y subvencionados de la ciudad de Talca. Noviembre 20, 2015, de SCIELO. Sitio web: http://www.scielo.cl/scielo.php?script=sci_arttext\&pi $\mathrm{d}=\mathrm{S} 0717-75182010000400002$

Rodríguez O., Tous M., Gil B., Longo G., Pereira J., García P. (2013). Impacto de una intervención educativa breve a escolares sobre nutrición y hábitos saludables impartida por un profesional sanitario. Septiembre 20, 2016, de SCIELO. Sitio web: http://scielo.isciii.es/scielo.php?script=sci_arttext\&pi $\mathrm{d}=\mathrm{S} 0212-16112013000500030$ 
Théodore F., Bonvecchio A.,Blanco I.,Irizarry L.,Nava A., Carriedo A. (2011). Significados culturalmente construidos para el consumo de bebidas azucaradas entre escolares de la Ciudad de México. Septiembre 20,

2015, de SCIELO Sitio web: http://www.google.com.mx/url?sa=t\&rct=j\&q=\&esrc $=\mathrm{s} \&$ source $=$ web \&cd=4\&ved $=0$ ahUKEwjogdP419TQ

AhXi5IMKHVSvD7YQFggqMAM\&url=http $\% 3 \mathrm{~A} \% 2$ F\%2Fwww.scielosp.org\%2Fpdf\%2Frpsp\%2Fv30n4\% 2Fv30n4a06.pdf\&usg=AFQjCNFSeAXPdFdEkcuVF wGmeYHMx_MElg\&sig2=NJk9qeH0_GrNWaTzZvIcg\&bvm=bv.139782543,d.amc

Velasco, A., Hernández, P. \& Aguilar, P. (2015) Estrategias de mercadotecnia de la industria de bebidas azucaradas. Instituto Nacional de Salud Pública. Sitio web: $\quad$ https://www.insp.mx/epppo/blog/3811estrategias-industria-bebidas.html 